\title{
EFFECTS OF GRAIN BOUNDARY MORPHOLOGY AND DISLOCATION SUBSTRUCTURE ON THE CREEP BEHAVIOR OF UDIMET 710
}

\author{
Y. Zhang ${ }^{+}$and F.D.S. Marquis* \\ +Department of Metallurgy, Chongqing University, P.R. China \\ *College of Physical, Chemical, and Materials Science and Engineering, \\ South Dakota School of Mines \& Technology, Rapid City, SD 57701, USA
}

\begin{abstract}
$\underline{\text { Abstract }}$
An extensive investigation of the effects of grain boundary morphology (GBM) and dislocation substructure (DS) on the creep behavior of a Udimet 710 alloy was carried out. The interactions between: (1) dislocations and transgranular particles, and (2) dislocations and grain boundary carbides were also investigated. A significant understanding of the strengthening mechanisms and the mechanisms of fracture in this alloy was achieved. Two types of specimens, one with straight grain boundaries (SGB) and the other with zigzag grain boundaries (ZGB), were obtained by specially designed thermomechanical processes. Specimens of each of these types were creep tested at $1123 \mathrm{~K}$ under an initial stress of $35 \mathrm{~kg} / \mathrm{mm}^{2}$.

Creep in a grain was primarily caused by dislocation climbing over $\gamma^{\prime}$ particles. The migration of grain boundaries was controlled by their gliding for the specimens with straight grain boundaries. However, for specimens with zigzag grain boundaries, creep in a grain was caused by both dislocations climbing over and dislocations cutting through $\gamma^{\prime}$ particles. The migration of grain boundaries was controlled by both gliding of grain boundaries and the "straightening" motion of grain boundaries in zigzag steps. Zigzag grain boundaries increased the crecp rate in a grain and decreased the gliding rate of grain boundaries. This effectively retarded the coalescence and growth of cracks and greatly enhanced the fracture resistance in the tertiary stage of creep observed at constant load. The creep life and plasticity to failure were increased in specimens with zigzag boundary morphology.
\end{abstract}

\section{Introduction}

It has been pointed out that zigzag grain boundaries obtained by special heat treatment techniques (involving slow cooling through the $\gamma^{\prime}$ solvus) could enhance the elevated and high temperature strength properties for most superalloys (1-6). Among those properties were the retardation of creep fracture processes and increments in creep failure plasticity of alloys (7).

No systematic studies of the effect of zigzag grain boundaries on dislocation substructures in creep, on creep

$$
\text { Superalloys } 1996
$$

Edited by R. D. Kissinger D. J. Deye, D. L. Anton,

A. D. Cetel, M. V. Nathal, T. M. Pollock, and D. A. Woodford

The Minerals, Metals \& Materials Society, 1996 resistance, and on fracture processes have been reported. Thus, in order to determine the fracture model and the strengthening mechanism of this alloy, it is very important to study the interactions between dislocations and $\gamma^{\prime}$ particles within a grain or dislocations and carbides (or other particles) at grain boundaries.

\section{Materials and Experimental Procedures}

The composition of the Udimet 710 alloy investigated is shown on table 1 .

Table 1. Composition of Udimet 710 Alloy (WT\%)

\begin{tabular}{llllll}
\hline & & & \\
$\mathrm{Ni}$ & $=$ & 57.77 & $\mathrm{~V}$ & $=$ & .26 \\
$\mathrm{Co}$ & $=$ & 14.77 & $\mathrm{Fe}$ & $=$ & .19 \\
$\mathrm{Cr}$ & $=$ & $\mathrm{C}$ & $=$ & .053 \\
$\mathrm{~W}$ & $=$ & 5.34 & $\mathrm{P}$ & $=$ & .006 \\
$\mathrm{Mo}$ & $=$ & 5.20 & $\mathrm{~S}=003$ \\
$\mathrm{Al}=$ & 3.79 & $\mathrm{~Pb}$ & $<$ & .001 \\
$\mathrm{Ti}$ & $=$ & &
\end{tabular}

Two types of specimens were produced in this alloy, those with zigzag grain boundaries and those with straight boundaries. Zigzag grain boundaries were obtained by the following isothermal treatments: $1473 \mathrm{~K} / 3 \mathrm{~h} / \mathrm{A} . \mathrm{C} .+1343$ K/ 3h/ A.C. +1123 K/ 8h/ A.C. Straight grain boundaries were obtained by conventional heat treatments: $1473 \mathrm{~K}$ / 2 h $/$ A.C. +1323 K/ 4h/ A.C. +1123 K/ 8h/ A.C., where A.C. means air cooling. A flow chart of these thermal proceses is shown on figure $1(a)$ and (b).

Specimens of each kind of these two different types of grain boundary morphologies were creeped at $1123 \mathrm{~K}$, at initial stress of $35 \mathrm{kgf} / \mathrm{mm}^{2}$.

Specimens for transmission electron microscopy (TEM) were prepared from $0.3 \mathrm{~mm}$ thick slices cut with an electric spark cutting machine from the creeped specimens. The specimens were subsequently ground to $0.03 \mathrm{~mm}$ and electrolytically thinned at $263 \mathrm{~K}$ using an electrolyte consisting of $10 \%$ perchloric acid and $90 \%$ ethanol mixture. The thin foils were examined using a JEM-1000 electron microscope operated at $1000 \mathrm{KV}$. 

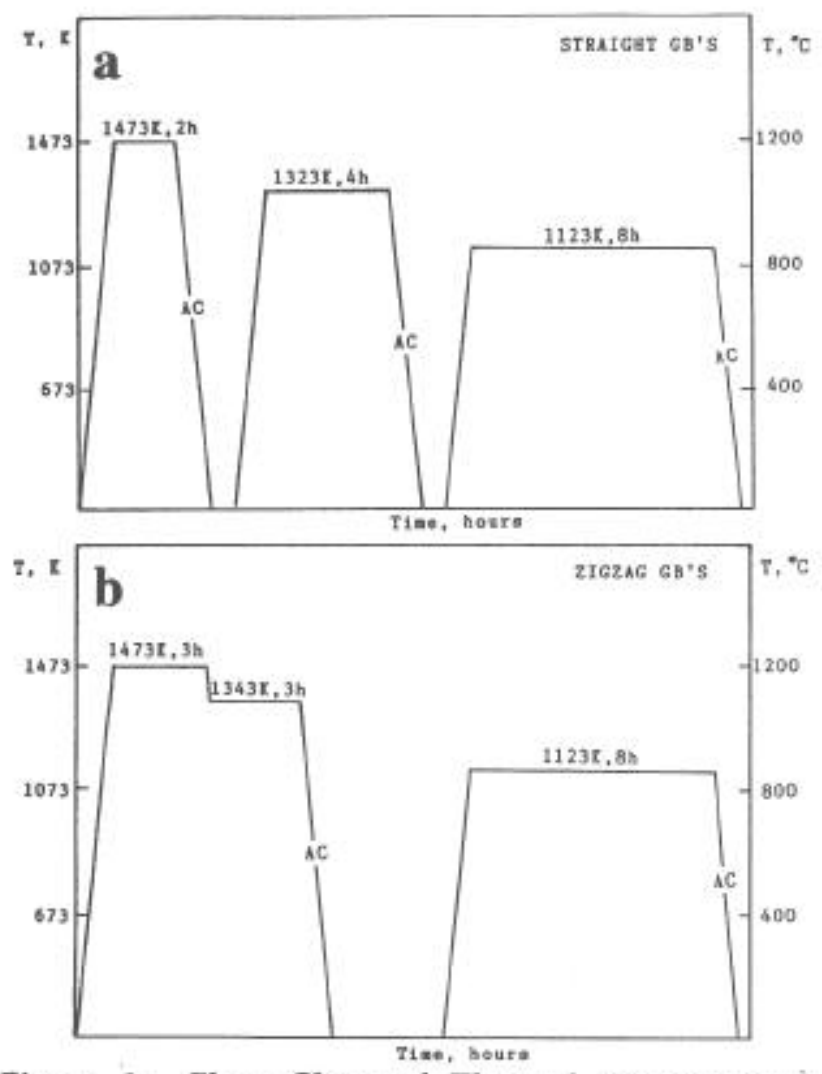

Figure 1: Flow Chart of Thermal Processing: (a) conventional (SGB) and (b) isothermal (ZGB).

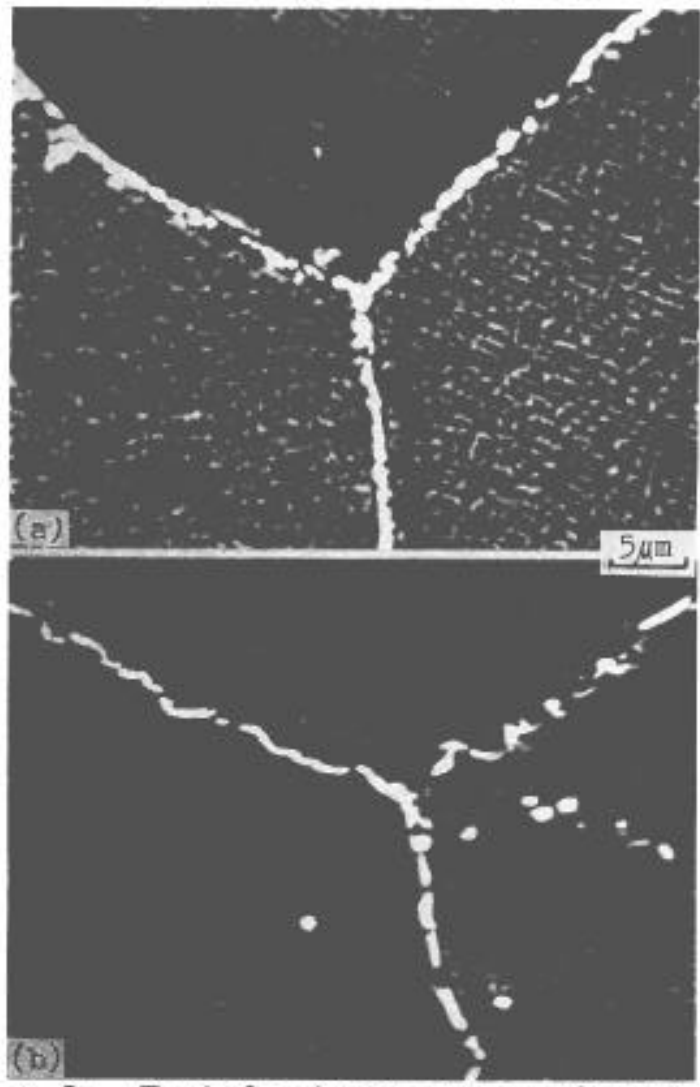

Figure 2: Typical microstructures after thermal processing: (a) straight GBs, (b) zigzag GBs.

\section{Results}

\subsection{Effect of Zigzag Grain Boundaries on Creep Life}

Typical microstructures obtained before creep are shown on figure 2. Figure 3 shows creep curves of specimens
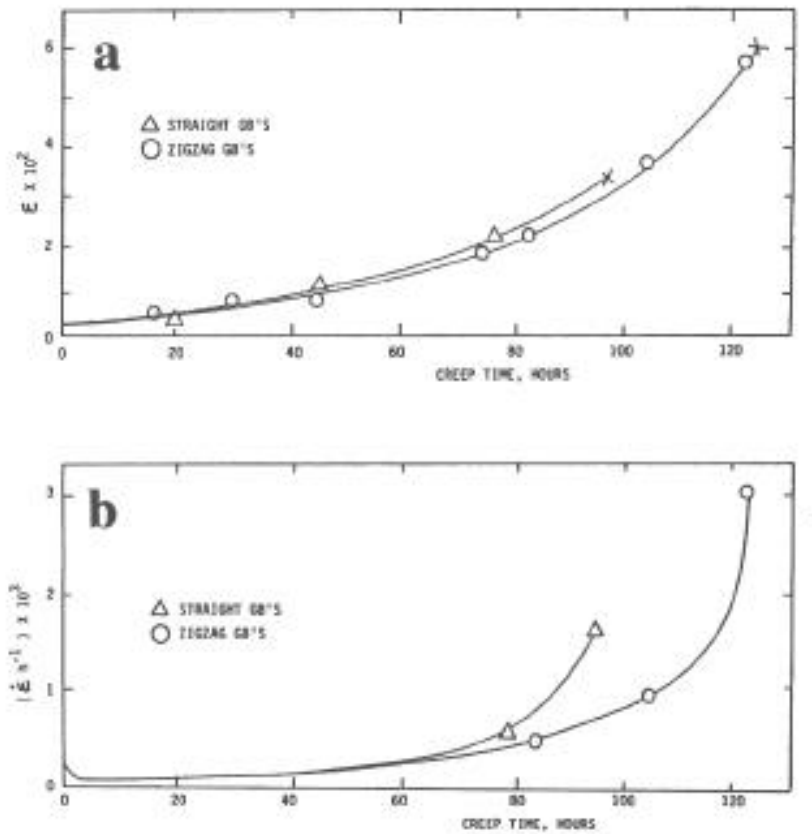

Figure 3: Creep behavior at $1123 \mathrm{~K}$ under an initial stress of $35 \mathrm{~kg} / \mathrm{mm}^{2}$ : (a) creep strain, (b) creep strain rate.

with different grain boundaries after being pulled to fracture. Typical microstructures of these specimens are shown on figure 4.

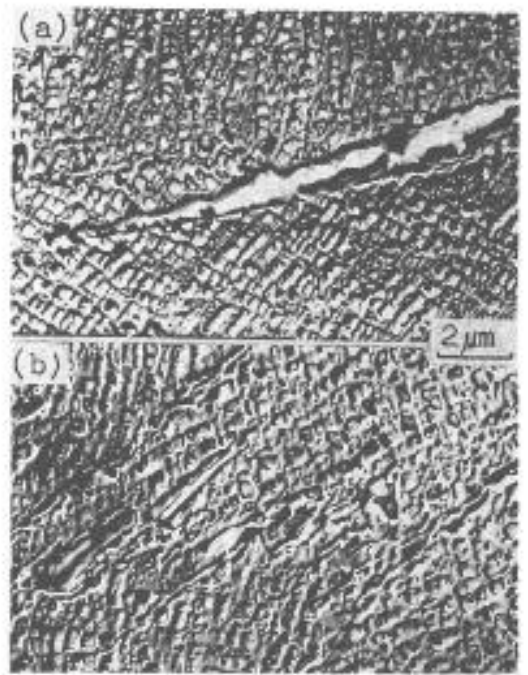

Figure 4: Typical microstructures after creep rupture: (a) straight GBs, (b) zigzag GBs.

The results show that zigzag grain boundaries lengthen the time of the tertiary stage of creep. The general creep 
life to failure increases by $26 \%$ and the creep plasticity by $73 \%$ in specimens with zigzag grain boundaries. The average life span and percentage of elongation increases
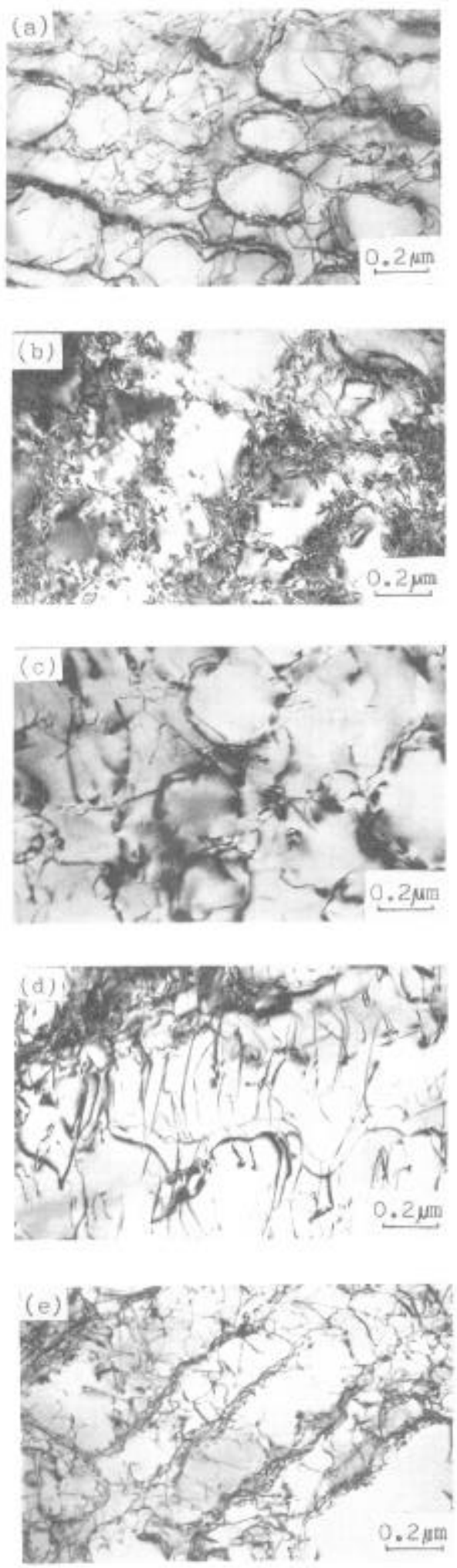

Figure 5: Dislocation substructures in grains with straight GBs: (a) 19.3h, (b) $44.8 \mathrm{~h}$, (c) and (d) $79.7 \mathrm{~h}$, (e) $94 \mathrm{~h}$. about $39.3 \%$ and $49.5 \%$, respectively, at $1173 \mathrm{~K}$ under 22 $\mathrm{kgf} / \mathrm{mm}^{2}$.

\subsection{Iranscrystalline Dislocation Substructures}

The dislocation substructrures within grains are illustrated in figure 5 (a), (b), (c), (d), and (e) for specimens with straight grain boundaries.

It was observed that during the creep process dislocations moved mainly within the $\gamma^{\prime}$ matrix. Many threedimensional dislocation networks and dislocation configurations after climbing over $\gamma$ particles existed at $\gamma / \gamma^{\prime}$ interfaces. The dislocation density increased with increasing creep time in all specimens with straight grain boundaries. No traces were observed which showed that dislocations cut through $\gamma^{\prime}$ particles. However at $\gamma / \gamma^{\prime}$ interfaces regular two-dimensional dislocation networks were clearly seen as shown in figure 5 (d).

Figure 6 shows the dislocation substructures of specimens with zigzag grain boundaries obtained during creep stages.

Observation revealed that the dislocations in the interfaces between cubic-shaped $\gamma^{\prime}$ particles and between small spherical $\gamma^{\prime}$ particles could be simulataneously observed when suitable orientation of grains and electron diffraction conditions were chosen (figure 6 [a]).

Many three-dimensional networks of dislocations were observed at $\gamma / \gamma^{\prime}$ interfaces and the dislocation density was higher at the interfaces between small spherical $\gamma^{\prime}$ particles. Trace analysis showed that dislocations cut through some cubic-shaped $\gamma^{\prime}$ particles. Other dislocations were observed to climb over $\gamma^{\prime}$ particles by a mechanism controlled by diffusion. At some reflection conditions it could be observed that dislocation couples exist in ' $\gamma^{\prime}$ particles, as illustrated by arrows in figure 6 (a) and (e). However, due to the high antiphase boundary energy, the space between two dislocations of a dislocation couple was too short to distinguish them on some crystallographic planes. As a result, only a slightly wider dislocation line could be observed, as illustrated by arrows in figure $6(\mathrm{~b})$ and (d). In addition, the increase of dislocation density was much larger than that in specimens with straight grain boundaries. These specimens exhibit lengthening of creep time, especially in the tertiary stage of creep. This can be well understood by comparing figures 2 and 3 .

For specimens with zigzag grain boundaries, changes in configurations of dislocation substructures show that the creep in a grain is controlled by both dislocation climbing over and cutting through $\gamma^{\prime}$ particles, at the beginning of the tertiary stage of creep. The transcrystalline deformation rate within grains was larger than that for specimens with straight grain boundaries. Cracks in the grain boundaries had initiated at the beginning of the tertiary creep. Even at later tertiary stage of creep, the deformation in grains still dominated the creep of the alloy. 

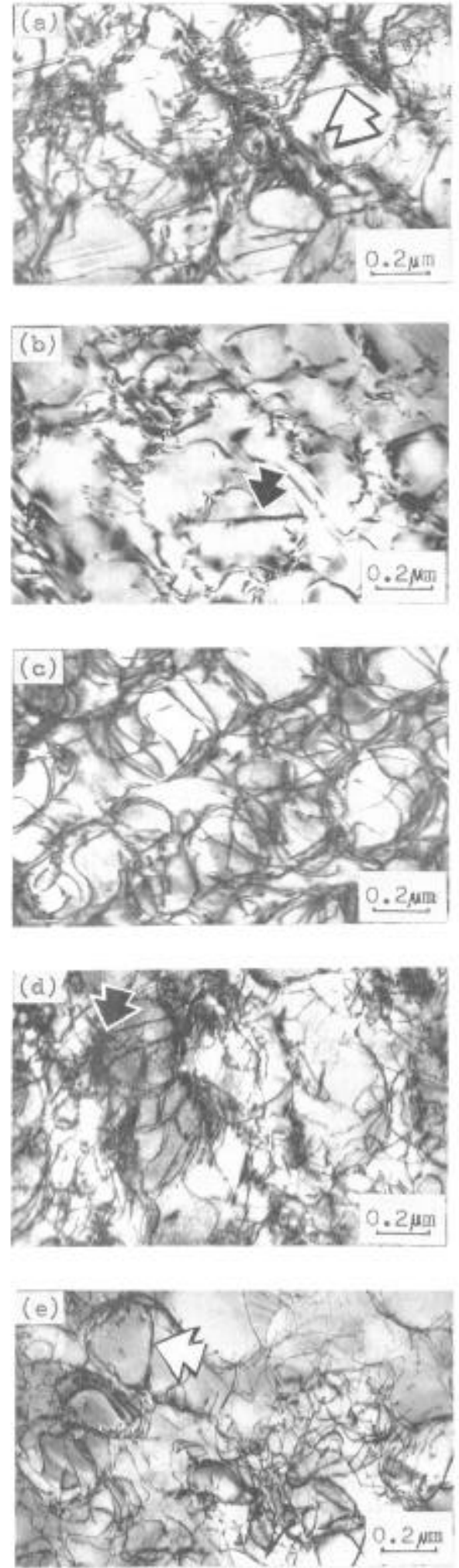

Figure 6: Dislocation substructures in grains with zigzag GBs: (a) $17 \mathrm{~h}$, (b) $43.8 \mathrm{~h}$, (c) $73.3 \mathrm{~h}$, (d) $117.3 \mathrm{~h}$, (e) $120 \mathrm{~h}$.

\subsection{Dislocation Substructures at and Near Grain Boundaries.}

For specimens with straight grain boundaries, the carbide morphology was mainly transcrystalline, although some $\mathrm{M}_{6} \mathrm{C}$ needle carbides were distributed at boundaries at the early stage of creep, as illustrated in figure 7 (a) (7-8).
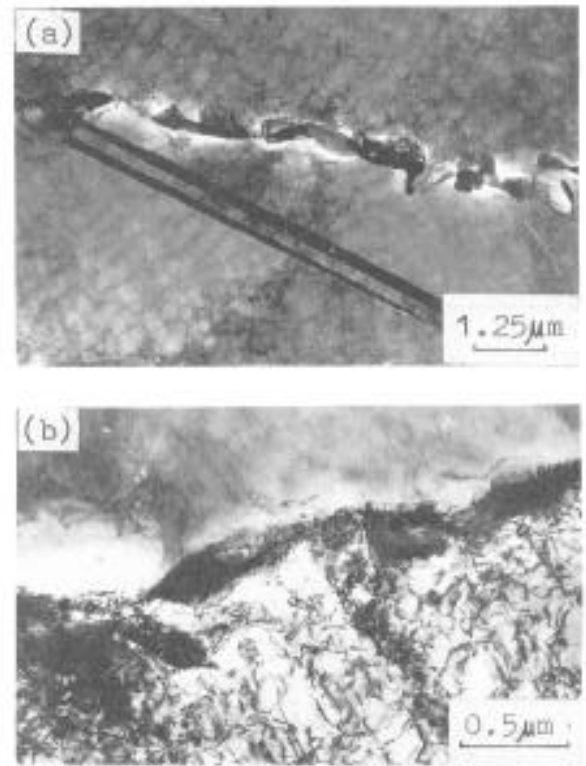

Figure 7: Dislocation substructures at and near GBs for specimens with straight GBs after creep for $19.3 \mathrm{~h}$.

Figure $7(b)$ shows that in specimens with straight grain boundaries, after creep for $19.3 \mathrm{~h}$ (still under conditions of steady state creep), a very large number of dislocation pileups were observed in regions near grain boundaries. Thus a few isolated cracks could be observed by means of optical microscopy at a few of these interfaces (figure 8).

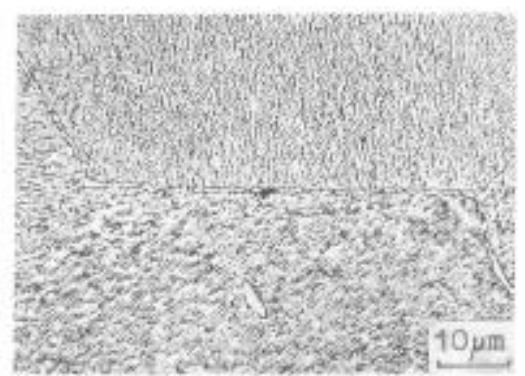

Figure 8: Crack at GB for specimen with straight GBs creeped for $19.3 \mathrm{~h}$.

It was observed that with the lengthening of creep time, the carbides distributed along longitudinal grain boundaries (parallel to the external force) move forward to transverse grain boundaries (perpendicular to the external force). This is consistent with other observations (9). After moving, the carbides distributed at transverse grain boundaries became strip-shaped. The amount of 
carbides increased and the space between carbide particles decreased as shown in figure 9.
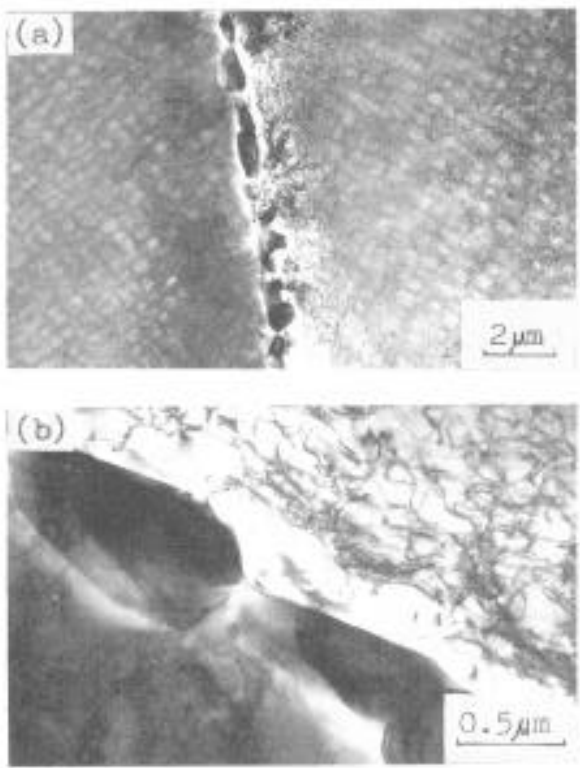

Figure 9: Dislocation substructures at and near GBs for specimens with straight GBs creeped for $79.7 \mathrm{~h}$.

The dislocation density near grain boundaries decreases in the tertiary stage of creep as shown by the comparison of figure $9(\mathrm{~b})$ with figure $7(\mathrm{~b})$. This process of dislocation relaxation is due to mechanisms occurring at dislocation pileups near grain boundaries. The head dislocations in the pileups climb to grain boundaries, which results in sliding of these grain boundaries. In these processes the unmatching displacements of sliding boundaries cannot be accommodated by transcrystalline microplasticity (i.e., by fractional quantities of dislocation motion within grains). Meanwhile, the carbides distributed at transverse grain boundaries have almost joined into thick plates covering all the interfaces, which provides a low energy path for crack growth. As a result, cracks grew so rapidly that specimens fractured. Figure 10 clearly shows the shape of the interfaces and the $\mathrm{M}_{6} \mathrm{C}$ carbide morphology, i.e., their size, interparticle spacing, and tri-dimensional distribution.

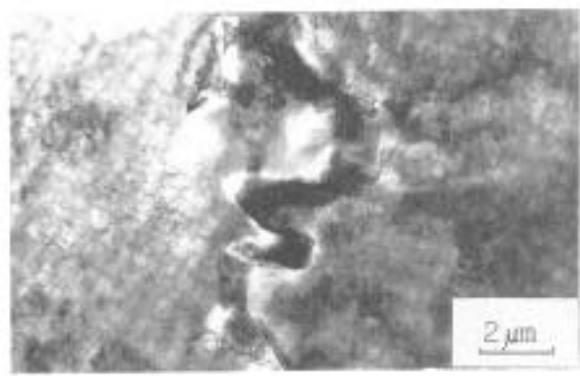

Figure 10: Morphology of zigzag GBs.

Thus, we can evaluate the changes occurring at grain boundaries. Specimens with straight boundaries and specimens with zigzag grain boundaries have different interfacial conditions. Therefore, the migrating and the precipitating of carbides at grain boundaries and the alternation of dislocation substructures near interfaces must be different during creep process.
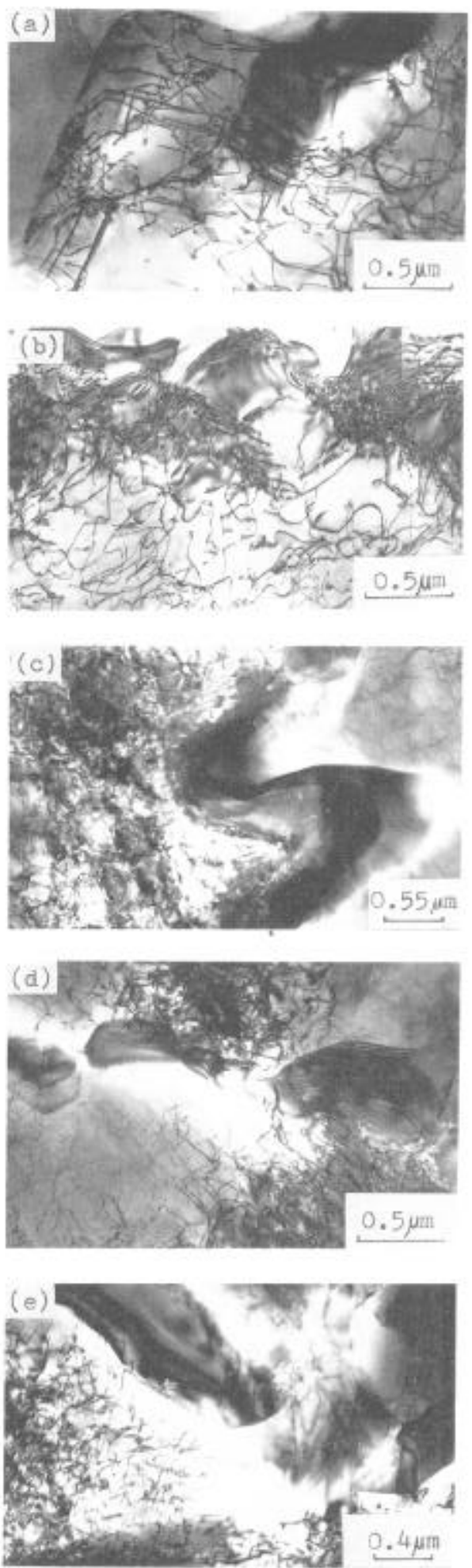

Figure 11: Dislocation substructures at and near interfaces in specimens with zigzag GBs: (a) $17 \mathrm{~h}$, (b) $43.8 \mathrm{~h}$, (c) $73.3 \mathrm{~h}$, (d) $117.3 \mathrm{~h}$. 
Figure 11 shows the structure of zigzag grain boundaries and the configurations of dislocations substructures near interfaces. It was observed that they have the following characteristics, as compared with straight interfaces:

1. With the lengthening of creep time, the wavelength $(\lambda)$ of zigzag grain boundaries increases and amplitude (h) decreases. This is in agreement with previous work (7). Meanwhile, the dislocation density near interfaces becomes heavier. On the other hand, the dislocation density near straight interfaces decreases with lengthening of creep time.

2. For stable conditions of creep and specimens creeped simultaneously, the dislocation density in regions near zigzag grain boundaries is lower than that near straight ones (figure 11[a] and figure $7[\mathrm{~b}]$ ). However, during the fast accelerating period of the tertiary stage of creep, not only is the dislocation density in regions near zigzag grain boundaries much heavier than that near straight ones, but dislocation networks become finer as well (figure 11[c], [d], and figure $9[\mathrm{~b} \mid)$.

3. With the lengthening of creep time, the curvature of zigzag grain boundaries $(h / \lambda)$ decreases, and the migration of carbides from vertical grain boundaries to transverse ones occurs mainly in zigzag steps of an interface. This makes coarse, irregular, bar-shaped carbides space themselves and become chain-shaped. This is very noticeable in late creep.

\section{Discussion}

\subsection{Interactions Between Dislocations and $\gamma^{\prime}$ Precipitates Within a Grain.}

After climbing over $\gamma^{\prime}$ precipitates, dislocations in a grain continue to move in matrix at $1123 \mathrm{~K}$, under a stress of 35 $\mathrm{kg} / \mathrm{mm}^{2}$ for specimens with straight grain boundaries. Dislocations at $\gamma / \gamma^{\prime}$ interfaces were of the type $a / 2<110>$ and during creep some of these dislocations moving on different slip systems react with each other. This gives origin to the formation of three-dimensional networks of dislocations that could be clearly seen at $\gamma / \gamma^{\prime}$ interfaces. While dislocations climb successively over $\gamma^{\prime}$ precipitates to continue motion, the dislocation density in grains continues to increases. However, at the stable creep stage, more networks of two-dimensional dislocations appear at $\gamma / \gamma^{\prime}$ interfaces (figure 12).

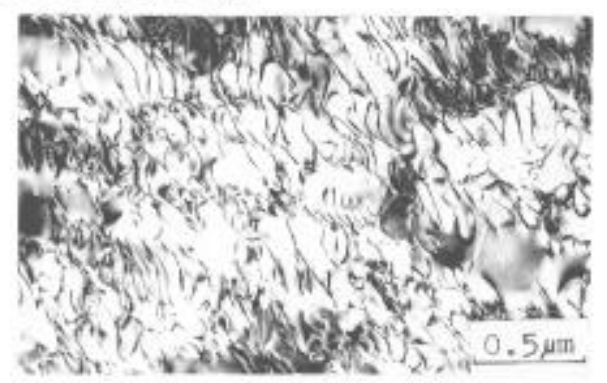

Figure 12: Regular networks of transgranular dislocations in ST creep for specimens with straight GBs (19.3h).
It is very possible that two groups of dislocations of type a $/ 2\langle 110\rangle$ react easily with each other at $\gamma / \gamma$ interfaces so that the dislocations are arranged evenly. This must result in dislocations climbing over $\gamma^{\prime}$ precipitates. In this process, the sliding distance on new slip planes of those dislocations after climbing becomes limited. This is not favorable to the deformation within grains.

It was observed that the coarsening of spherical $\gamma^{\prime}$ particles within grains and the decreasing of their volume fraction are much faster in specimens with straight grain boundaries than in specimens with zigzag grain boundaries. Consequently, the friction stress of dislocation motion within grains decreases rapidly. This is another reason why the climbing rate of dislocations increases. During the late tertiary stage of creep, the fine spherical $\gamma^{\prime}$ particles within grains were observed to coarsen rapidly and the climbing rate of dislocations was observed to increase. However, the free space of dislocation gliding was still limited by the regular twodimensional networks of dislocations which limited the creep deformation within grains.

For specimens with zigzag grain boundaries, the dislocations within grains not only could climb a great distance over $\gamma^{\prime}$ particles but could also cut through cubicshaped $\gamma^{\prime}$ particles to move continuously. This is clearly related to the deformation of cubic-shaped $\gamma^{\prime}$ particles.

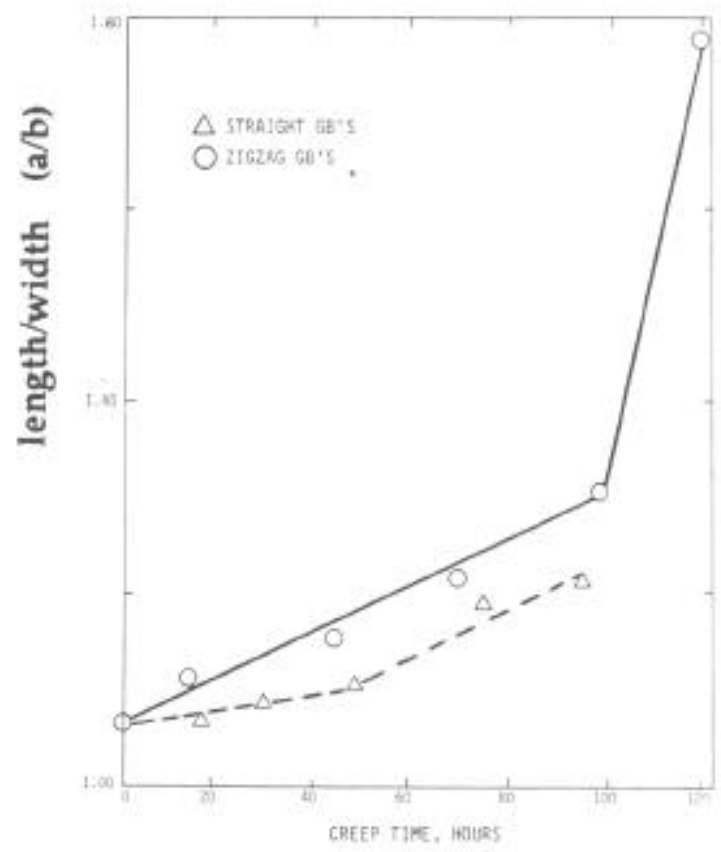

Figure 13: Effect of creep time on the length/width ratio (a/b) of $\gamma^{\prime}$ particles.

As shown in figure 13 the length/width ratio $(a / b)$ of 
cubic-shaped $y^{\prime}$ precipitates in specimens with zigzag grain boundaries is larger than in specimens with straight ones. Specifically, during the late tertiary stage of creep it is very clear that the former length/width ratio rises rapidly. However, the general volume fractions of cubic-shaped $\gamma^{\prime}$ precipitates is slightly lower than in specimens with straight grain boundaries. The interactions between dislocations and lengthened cubic-shaped $\gamma^{\prime}$ precipitates makes it possible for the dislocations to climb over many more $\gamma^{\prime}$ particles in order to continue motion. For specimens with zigzag grain boundaries, the motion of dislocations climbing in matrix and dislocations cutting through the $\gamma^{\prime}$ particles alternates. This results in (1) significantly higher dislocation density at $\gamma / \gamma^{\prime}$ interfaces than that in specimens with straight grain boundaries for the same creep time, and (2) the increase in the gliding distance on a new slip plane of those dislocations after climbing. In addition, there exists no inhibition to motion from regular two-dimensional networks of dislocations. This means that the free space of dislocation gliding is longer. Thus, the deformation in grains is much larger in specimens with straight grain boundaries. Even in the fast accelerating period of tertiary stage of creep, the deformation still primarily occurred within grains.

The present results show that the change in the grain boundary microstructure results in the change of creep mechanism in a grain. Dislocation climbing dominates for specimens with straight grain boundaries. Both dislocation climbing and cutting are important for specimens with zigzag grain boundaries.

\subsection{Characteristics of Zigzag Grain Boundary Motion. Effect on Dislocation Substructure.}

Because of the difference of boundary conditions, the motion characteristics of zigzag grain boundaries are different from those of straight boundaries. The present results show that the amplitude (h) of zigzag grain boundaries decreases and the wavelength $(\lambda)$ increases with the lengthening of creep time. This means that the migration of zigzag grain boundaries is characterized by boundary "straightening," which results in a "reversed" migration from zigzag grain boundaries to straight grain boundaries. The distortion field gives rise to additional resistance to dislocation motion. For the same creep period the rate of dislocation climbing forward to a grain boundary and the rate of dislocation sliding in specimens with zigzag grain boundaries is lower than that in specimens with straight grain boundaries. This is why a zigzag grain boundary retards cavity initiation. Thus, the motion of a zigzag grain boundary is characterized not only by the gliding of the grain boundary, but also by boundary "straightening." However, the motion of a straight boundary is primarily characterized by the gliding of the boundary.

During the tertiary stage of creep, the sliding rate (U) for specimens with zigzag grain boundaries was observed to follow the Raj and Ashby equation (13):

$$
\mathrm{U}=\alpha \mathrm{Mh}^{2}
$$

Since $\lambda$ increases with decreasing $h$, this must result in the increase of sliding rate (U) of a zigzag boundary in the late tertiary stage of creep. As a result, the initiation of cavitation becomes much easier. It is very interesting to notice that during the tertiary stage of creep, while the sliding rate of a zigzag grain is accelerating, the dislocation density near a boundary increases and the dislocation networks were finer than that near a straight one. These might be related to the deformation of grains and the migration characteristics of grain boundaries. Maclean (14) pointed out that the ability of alloys to bear external stresses is mainly determined by their dislocation density and dislocation distribution. This is consistent with the formation of higher density dislocation networks near zigzag grain boundaries. While the initiation of cavities or isolated cracks makes the area bearing external stresses decrease, the specimen with zigzag grain boundaries can bear greater stresses than those with straight grain boundaries. Thus, the stress factor of creep is increased. On one hand this shows that the sliding of zigzag grain boundaries is accommodated by the motion of dislocations which are produced step by step from grains. On the other hand, the motion of zigzag grain boundaries changes the configurations of dislocation substructures as a result of the creep resistance to damage being effectively enhanced.

It is known from the above that both the grain boundary sliding accommodated by creep mechanisms of dislocation climbing in a grain, as well as dislocation cutting and the "reversed" motion of a zigzag grain boundary, make the stress concentration caused by grain boundary sliding relax so that the coalescence and the growth of cracks becomes difficult. This is the essential reason why creep life increases.

\subsection{Interactions Between Dislocations and Grain Boundary Carbides.}

Carbides, which are mostly coarse, irregular-shaped particles, became distributed at concave or raised positions and stretched out along a zigzag grain boundary. The formation of zigzag grain boundaries is believed to decrease considerably the free energy of the system, thus making the zigzag grain boundary a low energy interface. The interactions between dislocations and coarse, irregular carbides make the activation of dislocation sources in a boundary become more difficult. Meanwhile, they might raise the creep activation energy of the atoms in the boundaries and decrease the diffusion coefficient of the atoms in the boundaries so that the creep stress factor is raised. This makes the migration rate of carbides decrease from vertical boundaries to transverse ones, and the motion of the carbides becomes limited at each short zigzag step in a zigzag grain boundary. As a result the coarse, irregular carbides develop into chain shapes (figure 14).

The small carbide sizes and the small spacing between neighbor carbides is believed to weaken the grain boundary. In spite of this, while the carbides in zigzag 
grain boundaries migrate, the diffusional path of atoms is limited in a short close step in the grain boundary. In addition, the chain-shaped carbides existing at transverse grain boundaries can retard boundary sliding. This effectively retards crack growth. During tertiary creep, the curvature $(h / \lambda)$ of zigzag grain boundaries decreases very rapidly, the atomic diffusional path lengthens, and the gliding rate of a boundary increases very rapidly. These cracks reach unstable conditions and grow rapidly, which results in final fracture.

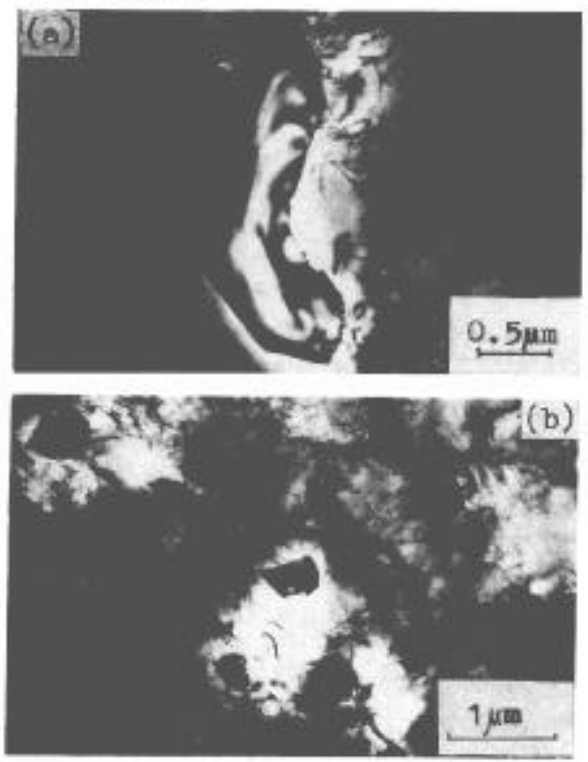

Figure 14: Carbide morphologies at zigzag GBs: (a) 17h, (b) 73.3h.

\section{Summary of the Behavior of Zigzag and Straight GBs.}

The present results, together with an ongoing investigation (15), show that the most significant differences in the behavior of specimens with zigzag grain boundaries versus those with straight grain boundaries are the following: (1) With increasing creep time, the wavelength $\lambda$ of zigzag grain boundaries increases, the amplitude $h$ decreases, and the adjacent dislocation density increases. The dislocation density adjacent to straight grain boundaries decreases with increasing creep time; (2) For steady state creep and specimens creeped simultaneously, the dislocation density in regions near zigzag grain boundaries is larger than that near straight grain boundaries. During tertiary creep, in regions near zigzag grain boundaries, the dislocation densities become much higher and the dislocation networks become finer than those near straight grain boundaries; (3) With increasing creep time, the curvature of zigzag grain boundaries $h / \lambda$ decreases and the migration of carbides from longitudinal grain boundaries to transverse grain boundaries occurs mainly in zigzag steps of the interface. This makes coarse irregular bar-shaped carbides space themselves and become chain-shaped. This is very noticeable in late creep.

\section{Conclusions}

1. Different morphologies of grain boundaries give rise to different creep mechanisms in a grain. Dislocation climbing over $\gamma^{\prime}$ precipitates dominates for specimens with straight grain boundaries. Both dislocation climbing over and cutting through $\gamma^{\prime}$ particles are important for specimens with zigzag grain boundaries. The interaction between dislocations and $\gamma^{\prime}$ precipitates force the bulk-grains to have a higher contribution for the deformation of specimens with zigzag grain boundaries.

2. Different morphologies of grain boundaries lead to different characteristics of grain boundary migration and different configurations of dislocation substructures. The migration of a straight grain boundary occurs mainly by boundary sliding. The migration of a zigzag grain boundary occurs mainly by boundary "straightening," i.e., a "reversed" migration from a zigzag grain boundary to a straight grain boundary.

3. Only a very small amount of grain boundary sliding was observed in specimens with zigzag grain boundaries. The dislocation density in regions near zigzag grain boundaries is higher and dislocation networks are finer than that near the straight ones, for the same time of tertiary creep stage. The sliding rate of a zigzag grain boundary is much lower than that of straight grain boundary. This becomes the key to enhance effectively the creep resistance and to lengthen the creep life of this superalloy,

4. The interactions between dislocations and coarse, irregular-shaped carbides at zigzag grain boundaries make the motion of carbides limited in each step of these boundaries. This presents the coalescence of carbides at traverse grain boundaries at early stages of creep and is very effective in retarding the crack growth and lengthening the time of the tertiary stage of creep. The creep life and plasticity to failure were increased in specimens with zigzag grain boundary morphology.

\section{References}

1. M. Yamazaki, Iournal of the Japan Institute of Metals 30 (1966), 1032.

2. M. Kobayashi et al., Iournal of the Iron and Steel Institute of Japan, 58 (1972), 859.

3. J. M. Larson and S. Floreen, Metall. Trans., 8 A (1977), 51.

4. Y. Ruizeng et al., Acta Metallurgica Sinica, 20 (1984), A34.

5. M. Zhiping et al., Research in Metallic Materials, 8 (1982), 5. 
6. G. Guixiong, Acta Metallurgica Sinica, 19 (1983),

A467.

7. Z. Yaping and L. Hong, Lournal of Chongqing University, 7 (1984), 141.

8. The Metallographic Diagram Collection of Superalloys, 1979, 134.

9. S. Kihara, J. B. Newkirk, A. Ohtomo, and Y. Saiga, Metall. Trans. 11A (1980), 1019.

10. W. Zhi, (Thesis, Dept. of Metallurgy, Chongqing University, 1984).

11. R. A. Sterens et al., Materials Science and Engineering, 50 (1981), 271.

12. L. Guoxon, The Principle of Physical Metallurgy, 306.

13. R. Raj and M. F. Ashby, Metall. Trans., 2 (1971), 1113.

14. D. Maclean, Rep. Prog. Phys., 29 (1966), 1.

15. F.D.S. Marquis, "Effects of Zigzag Grain Boundaries on Creep Behavior of Udimet 710," 121st TMS Annual Meeting, San Diego, CA, February, 1992. 